\title{
Quality Assurance in Regional Anesthesia: Current Status and Future Directions
}

\author{
Anjalee Brahmbhatt • Michael J. Barrington
}

Published online: 10 August 2013

(C) Springer Science + Business Media New York 2013

\begin{abstract}
Quality assurance in healthcare, including regional anesthesia is a large subject encompassing a range of elements. In this review article we aim to define the meaning of quality assurance and address how it can be achieved. The role of clinical registries in monitoring safety by collecting and analysing data from large patient cohorts and reporting outcomes is explored. Registries and similar multicentre studies in conjunction with incident reporting and root cause analysis provide the foundation for many quality improvement processes. Educating and training anesthesiologists to the highest standards is of paramount importance to delivering high quality care. The evolution of new technologies including ultrasound has significantly advanced our clinical practice, improving the safety and efficacy of peripheral nerve blocks. We review the role that guidelines, checklists, teamwork and procedure specific protocols play in the prevention of errors and safe delivery of regional anesthesia in the modern era.
\end{abstract}

Keywords Quality assurance $\cdot$ Healthcare $\cdot$ Regional anaesthesia $\cdot$ Nerve conduction $\cdot$ Education $\cdot$ Clinical registries

\section{Introduction}

The healthcare industry is a large, complex dynamic system that is hugely expensive. The system is not only comprised

\author{
A. Brahmbhatt $(\bowtie) \cdot$ M. J. Barrington \\ St Vincent's Hospital, 41 Victoria Parade Fitzroy, Melbourne, \\ Victoria 3065, Australia \\ e-mail:drab30@yahoo.com \\ M. J. Barrington \\ e-mail: michaelbarrington84@gmail.com
}

of the patient and patient's family, but is also a diverse range of health care workers, a complex array of equipment and technology and a never-ending list of new therapies. A range of administrative, political and cultural factors influence healthcare $[1 \bullet]$. The above factors are relevant to the specialty of anesthesia, for example, the work of an anaesthetist frequently interfaces with medical, surgical and nursing disciplines. In addition, the operating room can be a demanding workplace with production pressures and the physical environment presenting numerous challenges. Measuring quality and outcomes of care are particularly challenging as anesthesia is embedded into the overall perioperative care pathway where outcomes are often multifactorial. Regardless of these complexities and system issues, the public's perception of safety and anesthesia is primarily related to the rare risk of death due to general anesthesia.

Quality can be described as the general excellence of standard or the degree or grade of excellence of a trait or characteristic of a system. There is no single definition of quality, it is essentially non-measurable and is challenging to assess in the context of healthcare. In 1966, Donabedian developed a model to evaluate quality of healthcare and this has remained a dominant paradigm in this field. The model describes health care in terms of structure (staff, material, organisation), process (the activities) and outcomes (results of our care) [2•]. Information about the quality of care can be derived from all three elements. Quality of care can also be described using the following attributes: appropriateness, availability, continuity, effectiveness, efficacy, efficiency, prevention, respect and caring, safety, timeliness and overuse/underuse. Clinical indicators have been developed in an attempt to measure quality. However, clinical indicators used in anesthesia are often perioperative rather than anesthesia-specific and 
many indicators are processes of care developed from expert opinion rather than scientific evidence [3]. Clinical indicators rarely include patient views on the quality of care they received, however, obtaining feedback from patients can provide us with valuable insight into those elements of care that patients consider important [4]. In anesthesia, quality and safety are traditionally measured by the rate of adverse events [5] and this is a logical approach, given the invasive nature of the procedures we perform.

Quality assurance can be defined as ensuring clinical care is consistently practiced and delivered to the highest standards. Quality improvement has previously been defined as a combination of efforts by the multidisciplinary team in making the necessary changes that result in improved patient outcomes, improved system performance and enhanced professional development [6]. Increasing healthcare needs, pressures on service delivery and the advent of new technologies mean that maintaining the highest standards can be challenging.

Quality assurance and improvement can be achieved through a variety of methods, including monitoring and reporting the quality of care, evaluating our current standards of practice and comparing these to 'gold standards'; the use of protocols and guidelines, and continual educational and professional development. The need for quality assurance in regional anesthesia has led to the development of projects that have collected data on a large scale [7•]. Analysis of this data has been used to identify areas where clinicians can improve their practice and implement improvement strategies. Similarly, critical incident reporting and root cause analysis are imperative to better our understanding of the cause of complications. This review will focus on key aspects of quality assurance and improvement including studies that have monitored the quality and safety in regional anesthesia, training and education; professional guidelines and patient satisfaction. Finally, we make some recommendations regarding future direction and priorities for quality assurance.

\section{Monitoring Quality and Safety in Regional Anesthesia}

Monitoring the quality and safety of regional anesthesia is of paramount importance for informed patient consent and clinical decision-making. The challenges of providing information on serious complications of regional anesthesia were revealed in a 2008 survey of the American Society of Regional Anesthesia and Pain Medicine (ASRA) members [8]. The survey respondents reported widely varying incidences of serious risks including nerve injury. This was proposed as being related to the existence of only a few studies on the subject and the methodological limitations including self-reporting [9] and reviews of insurance claims [10]. The authors of the survey commented on the importance of accurate numerical disclosure of risks for informed patient consent.

Postoperative nerve injury is often presumed to be a risk unique to regional anesthesia, however, there is good evidence that nerve injury associated with peripheral regional anesthesia is part of a larger subset of perioperative nerve injury with diverse etiologies [11, 12]. Fortunately, regardless of etiology, serious neurologic adverse events occur with relative rarity. However, an adverse event that occurs infrequently is still important to the patient and also in terms of how our colleagues and the public view our specialty. It is demanding to obtain reliable incidence data on events that occur infrequently and large patient cohorts are required. In a 2008 ASRA practice advisory on neurologic complications, Neal commented on how the relative rarity of complications made it difficult to obtain reliable incidence data and that: "randomized controlled trials and other tools of evidenced-based medicine hardly ever existed on the subject and that they would be unlikely to do so in the future" [13].

Monitoring the quality and safety of regional anesthesia is also important because clinical practice is continually evolving; examples of this include the shift from neuraxial to peripheral regional anesthesia and more recently the use of local infiltration analgesia. Clinical and technological advances have occurred including the widespread use of ultrasound imaging for peripheral nerve blockade (PNB) $[14,15]$. Nevertheless, new technology does not automatically equate with improved quality and safety. All new technologies, devices and drugs that patients are exposed to should be assessed for safety and effectiveness.

\section{Examples of Studies that Have Obtained Large Patient Cohorts}

Cook led a collaborative effort involving all United Kingdom (UK) National Health Service hospitals and reported that the incidences of paraplegia or death following neuraxial blockade were 1.8 and 0.7 per 100,000, respectively [16•]. Patients receiving perioperative epidural analgesia were at higher risk of complications. Moen performed a retrospective study on severe complications following neuraxial blockade in Sweden [17]. There were 127 complications with permanent neurologic damage in 85 patients, from a denominator of 1,260,000 spinal and 450,000 epidural anesthetics. More complications occurred in patients following orthopedic surgery and those receiving epidural anesthesia. The incidence of epidural hematoma ranged from 1:3,600 following total knee arthroplasty to $1: 200,000$ following obstetric epidural anesthesia. This finding demonstrated the impact that patient (e.g., lumbar 
canal stenosis) and surgical factors have on outcome. In a 1-year nationwide survey in Denmark, Wang et al. [18] estimated the incidence of epidural abscess to be $1: 1930$. Risk factors for abscess were immunosuppression and prolonged duration of catheterization ( $>5$ days). Delayed diagnosis of abscess was associated with poor neurologic outcomes. In a large population-based cohort study, the incidence of decompressive laminectomy was unchanged if patients received epidural $(8: 44,094)$ or general anesthesia (7:44,094) [19]. This incidence of laminectomy is similar to the abovementioned UK $(1: 8,100)[16 \bullet]$ and a singlecenter study $(1: 8,000)$ [20]. These studies consistently indicate that the incidence of serious complications following neuraxial anesthesia is rare.

Auroy used voluntary reporting to estimate the incidence of major complications of regional anesthesia in France [9]. From a total of 50,000 PNBs there were 12 neurologic complications, seven of which had sequelae at six months. In contrast, Capdevila reported an incidence of nerve injury of $0.21 \%$, a whole order of magnitude higher following continuous PNB. However, the symptoms of one of three patients with potential complications resolved within $72 \mathrm{~h}$ and the symptoms of the remaining two resolved by three months [21]. Orebaugh using analysis of a quality improvement database and billing records, reported an incidence of nerve injury following 9069 PNBs of $0.09 \%$ at six months [22]. Sites determined that the incidence of postoperative neurological symptoms persisting at six months following $12,668 \mathrm{PNBs}$ to be $0.09 \%$ [23]. Three studies from the Mayo Clinic following knee, hip and shoulder arthroplasty determined that the use of PNB did not increase the risk of postoperative nerve injury $[24,25 \cdot 26]$.

\section{The Role of Clinical Registries in Measuring Quality and Safety}

Registries are increasingly attracting the attention of funders, regulators and administrators as health care systems seek more information on quality and safety. Clinical registries systematically and uniformly collect information from people who undergo a procedure, are diagnosed with a disease or use a health care resource [27]. The American Heart Association defines a clinical registry as a prospective observational database of a clinical condition, procedure, therapy or population, in which there are no registry-mandated approaches to therapy and relatively few inclusion and exclusion criteria [28]. Clinical registries are increasingly important for monitoring and benchmarking the quality of clinical care. The assessment of safety in observational trials when sufficiently comprehensive and large, may offer critical insights, even in light of recognised limitations [29]. A key strength of a registry is the ability to sequentially enrol an entire patient population.

The Anesthesia Quality Institute, created by the American Society of Anesthesiologists, is an important data source and infrastructure for quality improvement processes in anesthesia [30]. The institute supports the National Anesthesia Clinical Outcomes Registry that has gathered data on millions of cases. Registry participants range from practices that record on paper documents to centers with sophisticated electronic systems. The Australian and New Zealand Registry of Regional Anaesthesia (AURORA), is a multicentre prospective clinical registry that monitors and reports on the quality and safety of PNB. AURORA utilizes a proactive approach to capturing complications that includes systematic postoperative contact with all patients. AURORA has demonstrated that serious adverse events associated with PNB are infrequent or rare. Importantly, AURORA has demonstrated that ultrasound guidance improves safety because it is associated with a reduced incidence of local anesthetic systemic toxicity (LAST) [31, 32].

In a recent review by Polaner, the authors stress the importance of generating data on a sizeable scale, to identify current standards of practice and enabling individual departments to compare themselves against peers [7]. A regional anesthesia network for pediatric patients was established to collate data on safety, complications, and risks associated with regional anesthetics in children. Databases that collate large numbers may have the ability of detecting small differences in practice, which could potentially result in improved patient outcomes.

\section{Technology and Equipment}

Meta-analyses have demonstrated improved efficacy following ultrasound-guided techniques compared to nerve stimulator techniques [33-35]. The chief utility of ultrasound-guided PNB is the ability to image nerves, nerve plexuses, needles, injectate and to avoid structures such as blood vessels. Ultrasound technology itself has rapidly evolved, with improved image resolution and ergonomics. The use of echogenic needles may increase the safety and efficacy of ultrasound-guided PNB [36]. Limitations still exist using two-dimensional ultrasound, as only a shortaxis or long-axis view is available at any particular moment in time. Multidimensional imaging using three-dimensional ultrasound may partially overcome these issues and be utilised in the future [37-39]. The anesthesiologist has a responsibility to ensure they understand the function of the equipment they use and to check it before use. It is well known that a range of clinical and professional behaviors are associated with the quality and success of regional 
anesthesia rather than reliance on one type of technology [40].

\section{Guidelines for Safe Conduct of Regional Anesthesia}

Guidelines for the safe conduct of regional anesthesia have been promulgated by professional organisations. The Australian and New Zealand College of Anaesthetists (ANZCA) publishes guidelines for the management of major regional anesthesia, patient consent, facilities, equipment, assistance, infection control and sedation. A lead anesthesiologist or director of regional anesthesia should be appointed in each department to provide support and supervision in regional anesthesia. Minimal standards of monitoring are deemed necessary during performance of a local or regional anesthetic technique. These include the application of non-invasive blood pressure monitoring, pulse oximetry and electrocardiography [41]. Skilled trained assistance should be present at all times [42]. Guidelines for the management of LAST have been produced by the American Society of Regional Anesthesia and Pain Medicine and the Association of Anaesthetists of Great Britain and Ireland [43, 44].

\section{Procedure Specific Protocols, Checklists and Teamwork}

Procedure specific protocols and checklists have a role in the prevention of errors and the safe delivery of regional anesthesia. Numerous protocols have been developed in an effort to standardize care and effectively manage patients in the perioperative period. Hebl et al. [45] reviewed 100 patients undergoing hip or knee surgery using the Mayo Clinic Total Joint Regional Anesthesia protocol and suggested that this protocol may improve outcomes such as reduced length of hospital stay. Protocols such as these may have significant economic implications reducing healthcare costs [46].

The concept of using checklists in surgical and anesthetic practice was endorsed following the 2008 publication of the WHO Surgical Safety Checklist [47]. It was believed that perioperative morbidity and mortality could be reduced by routinely checking common safety issues and improving team communication [48]. Accurate written or electronic records confirming completion of the checklist should be recorded, supplemented by a briefing. Haynes et al., prospectively collected data from over 3000 patients following introduction of the surgical safety checklist. The authors found that implementation of the checklist was associated with a reduction in the rates of death and complications in a diverse group of hospitals [49]. However, a recent study published by Haugen [50] demonstrated that successful implementation of a surgical checklist had limited impact on safety culture. The authors conducted a pre and post intervention survey of perioperative personnel including surgeons, anesthesiologists and anesthetic nurses. A validated tool was used to evaluate whether exposure to a checklist resulted in changes to personnel perceptions of safety culture. This finding differs considerably from a study by Allard [51], who found an association between briefing practices and attitudes towards safety.

Teamwork is increasingly recognised as being of paramount importance for high quality clinical care and one example is its use in the prevention of wrong-site PNB. A patient safety initiative was recently introduced at Nottingham University Hospitals, UK in association with Safe Anaesthesia Liaison Group. The initiative, titled 'Stop Before You Block', is an attempt to reduce the incidence of wrong-site PNB [52]. Recommendations published by the Safe Anaesthesia Liaison Group [53] suggested that the patient should be correctly marked by a member of the surgical team prior to performing the block, as per checklist guidelines. The report also found that a recurring cause of 'wrong-site' blocks included distraction in the anesthetic room, a time delay between performing the checklist and PNB; and patients being covered by blankets. The campaign suggests the 'stop before you block' moment can be instigated by any member of the anesthetic team highlighting the importance of teamwork and communication.

\section{Training and Education}

Quality issues can be embedded in methods used for professional formation and development. Training anesthesiologists to a high standard is integral to assuring quality. Ultrasound guided regional anesthesia (UGRA) requires the ability to simultaneously image a target nerve/plexus, needle trajectory, injectate and surrounding structures. Although there is evidence that ultrasound-guided techniques improve efficacy and block onset time, in the context of training, time pressure may be a significant problem. Organising the patient to arrive well in advance of the anticipated operating start time in an area physically separate to the operating room such as an anesthetic or block room may enhance the teaching environment.

The safe practice of regional anesthesia requires both knowledge and practical skills and correct identification of all anatomical structures in the procedural field is an important prerequisite [54]. Performing regional anesthesia without an appropriate knowledge of anatomy may be considered substandard, bordering close to negligence [54]. Recently published guidelines by the American and European Societies of Regional Anesthesia have encouraged quality improvement processes that include practicing 
ultrasound scanning techniques and working closely with experienced practitioners [55]. In a survey, Helwani et al. [56] found that most trainees were taught ultrasoundguided PNB.

Teaching in the clinical environment can be opportunistic and ad-hoc, with service delivery predominating. Relative irregularity of appropriate surgical cases combined with reduced working hours may further limit training opportunities. Various teaching methods have been employed to overcome these issues including the use of video teaching, part-task trainers and cadavers. Virtual reality (VR)-based simulators have been developed to teach regional anesthesia, however, VR simulators often fail to consider individual anatomy thereby reducing fidelity. Grottke et al. [57•] have developed a VR-simulator that can be used for individual anatomy for different anatomical regions. Rosenberg developed sonoanatomically based partial-task simulators to teach UGRA [57•]. The authors commented on the importance of developing educational tools that simulate real anatomy including sonoanatomy and tactile feedback. Moore established an educational programme for pediatric fellows using a combination of real-time simulation, cognitive skill assessment and feedback [58]. The authors found a significant improvement in the trainee's technical and cognitive skills at six and 12 months. High-fidelity simulation can be a way of training novices in managing emergencies including severe LAST. Neal et al. [59॰] assessed whether the use of the ASRA checklist would improve a trainees' ability to manage LAST. The authors concluded that using a checklist significantly improved the trainee's ability to manage the emergency using simulation. Cadavers are useful for training novices UGRA having realistic anatomy and tactile feedback [60]. We would strongly endorse the use of ultrasound scanning to teach trainees anatomy. Following appropriate patient consent, obtaining proficiency in sonography and recognising sonoanatomical landmarks can be taught using surgical patients independent of the performance of PNB.

Assessment of procedural skills during training is of importance to assess the quality of the final product. One method of procedural assessment is the Direct Observation of Procedural Skills (DOPS) tool comprising domains that assess procedural skills, attitudes, behaviours and knowledge [61]. A combination of various educational strategies including validated assessment tools and feedback can be utilised for quality improvement in training.

\section{Critical Incident Reporting and Risk Analysis}

An example of incident reporting is the Australian Incident Monitoring Study where participating anesthetists were invited to report, on an anonymous and voluntary basis, any unintended incident, which reduced, or could have reduced, the safety margin for a patient. This study provided detailed qualitative information such as the characteristics that underlie adverse events and was used to develop strategies to prevent and manage problems and to plan further initiatives [62]. The Anesthesia Quality Institute supports an Anesthesia Incident Reporting System that confidentially collects serious adverse events and near misses.

\section{Patient Satisfaction}

Patient satisfaction is an important outcome following regional anesthesia, although only a limited number of studies have been conducted on the subject. Patient rated outcomes and measuring their perceptions of care are alternatives to traditional measures of quality that primarily focus on outcomes that physicians consider important. A patient-centred approach enables clinicians to obtain a broader assessment of the different domains representing quality [63]. Perceptions of care are rarely incorporated in the development of clinical indicators or design of studies into quality and safety [3, 64]. However, Quality of Recovery (QoR) has been recognised as a useful tool in assessing quality. QoR-40 is a 40-item questionnaire assessing five dimensions: patient support, pain, comfort, emotions and physical independence. In a recent systematic review by Gornall, the authors have suggested that QoR-40 is a suitable measurement tool for postoperative QoR and patient satisfaction, including those patients who have received regional anesthesia [65].

Studies indicate that patients place significant importance on communication and information provided to them in the perioperative period [4]. Numerous professional bodies including the Royal College of Anaesthetists UK, and ANZCA emphasize the importance of providing patients with detailed written and verbal information regarding their anesthesia care [66].

\section{Future Directions}

Training and education forms the foundation for future quality of care in regional anesthesia. Therefore, utilization of specific educational strategies, rather than relying on volume of exposure and assessment of procedural skills using validated educational tools such as DOPS are likely to assume greater importance in our training programs. Systematic monitoring and reporting of outcomes using registries and similar multicentre methodologies are critical to describe and define our evolving practices. Outcomes should include a broad range of metrics and outcomes that define quality should include patients' perceptions of the 
care they received. Evaluation of new technologies and equipment will remain important. Collaboration using online interfaces connected to remote secure databases provide opportunities to develop a 'virtual department of anesthesiology' [67]. Independent of any electronic devices and interfaces, we should recognize that anesthesiologists' providing routine clinical care are our greatest quality improvement resource because of their potential to collectively capture quality data close to the point of care from large patient cohorts. In our quality improvement endeavours we should remain cognizant that the science of safety did not originate in healthcare and there is likely value in collaborating with quality and safety experts outside of the healthcare industry.

\section{Conclusion}

Acquiring data from large patient cohorts are often required to benchmark and describe the quality of care including the incidence of complications. Existing large scale and nationwide studies indicate that the incidence of serious adverse events attributable to regional anesthesia is infrequent or rare. However, ongoing evaluation of the quality of our care is important; hence, quality assurance and improvement are critical processes that all anesthesiologists should be actively involved in. Indeed, it is the responsibility of all clinicians to provide data from their routine practice so as to improve the quality of clinical practice. The initial training of anesthesiologists, assessment of the final product and subsequent continual professional development remain important for delivering high quality care. Protocols, checklists, teamwork and attitudes to safety in routine clinical practice are all essential components of the modern quality paradigm.

\section{Compliance with Ethics Guidelines}

Conflict of Interest Anjalee Brahmbhatt and Michael J. Barrington declare that they have no conflict of interest.

Human and Animal Rights and Informed Consent This article does not contain any studies with human or animal subjects performed by any of the authors.

\section{References}

Papers of particular interest, published recently, have been highlighted as:

- Of importance

1. - Vincent C, Batalden P, Davidoff F. Multidisciplinary centres for safety and quality improvement: learning from climate change science. BMJ Qual Saf. 2011;20 Suppl 1:i73-8. Epub 2011/04/ 06. The importance of multidisciplinary work of scholars and scientists from non-healthcare fields is emphasized for improving processes and outcomes in healthcare. Permanent multidisciplinary centres are required to provide sustained inspiration, research, training and practical support.

2. - James S, Frobert O, Lagerqvist B. Cardiovascular registries: a novel platform for randomised clinical trials. Heart. 2012. Epub 2012/05/18. The pros and cons of the methodology of randomized clinical registry trials are discussed including the ability to obtain large cohorts of consecutive patients to allow collection of infrequent events. The Swedish Coronary Angiography and Angioplasty Registry is used as an example of a successful registry.

3. Haller G, Stoelwinder J, Myles PS, McNeil J. Quality and safety indicators in anesthesia: a systematic review. Anesthesiology. 2009;110(5):1158-75.

4. Fung D, Cohen M. What do outpatients value most in their anesthesia care? Can J Anaesth. 2001;48(1):12-9.

5. Benhamou D, Auroy Y, Amalberti R. Monitoring quality and safety in anesthesia: are large numbers enough? Anesth Analg. 2008;107(5):1458-60.

6. Batalden PB, Davidoff F. What is "quality improvement" and how can it transform healthcare? Qual Saf Health Care. 2007; 16(1):2-3.

7. - Polaner DM, Martin LD. Quality assurance and improvement: the pediatric regional anesthesia network. Paediatr Anaesth. 2012;22(1):115-9. Describes a large-scale collaborative database used to establish standards of practice and define the incidence of complications. This network offers insight into how such data can be used to detect trends in adverse events and improve care.

8. Brull R, Wijayatilake DS, Perlas A, Chan VW, Abbas S, Liguori $\mathrm{GA}$, et al. Practice patterns related to block selection, nerve localization and risk disclosure: a survey of the American Society of Regional Anesthesia and Pain Medicine. Reg Anesth Pain Med. 2008;33(5):395-403.

9. Auroy Y, Benhamou D, Bargues L, Ecoffey C, Falissard B, Mercier FJ, et al. Major complications of regional anesthesia in France: The SOS Regional Anesthesia Hotline Service. Anesthesiology. 2002;97(5):1274-80.

10. Aromaa U, Lahdensuu M, Cozanitis DA. Severe complications associated with epidural and spinal anaesthesias in Finland 1987-1993. A study based on patient insurance claims [see comment]. Acta Anaesthesiol Scand. 1997;41(4):445-52.

11. Welch MB, Brummett CM, Welch TD, Tremper KK, Shanks AM, Guglani P, et al. Perioperative peripheral nerve injuries: a retrospective study of 380,680 cases during a 10-year period at a single institution. Anesthesiology. 2009;111(3):490-7.

12. Barrington MJ, Snyder GL. Neurologic complications of regional anesthesia. Curr Opin Anaesthesiol. 2011;24(5):554-60.

13. Neal JM, Bernards CM, Hadzic A, Hebl JR, Hogan QH, Horlocker TT, et al. ASRA Practice Advisory on Neurologic Complications in Regional Anesthesia and Pain Medicine. Reg Anesth Pain Med. 2008;33(5):404-15.

14. Gray AT. Ultrasound-guided regional anesthesia: current state of the art. Anesthesiology. 2006;104(2):368-73 discussion 5A.

15. Marhofer $\mathrm{P}$, Chan VW. Ultrasound-guided regional anesthesia: current concepts and future trends. Anesth Analg. 2007;104(5): $1265-9$, tables of contents.

16. - Cook TM, Counsell D, Wildsmith JA. Major complications of central neuraxial block: report on the Third National Audit Project of the Royal College of Anaesthetists. Br J Anaesth. 2009;102(2):179-90. Epub 2009/01/14. This recent successful nationwide collaboration provided a detailed appraisal of the rarity of serious risks associated with neuraxial anesthesia. The existence of this project highlights how regional anesthesia is scutinised for rare and disastrous events. 
17. Moen V, Dahlgren N, Irestedt L. Severe neurological complications after central neuraxial blockades in Sweden 1990-1999. Anesthesiology. 2004;101(4):950-9.

18. Wang LP, Hauerberg J, Schmidt JF. Incidence of spinal epidural abscess after epidural analgesia: a national 1-year survey. Anesthesiology. 1999;91(6):1928-36.

19. Wijeysundera DN, Beattie WS, Austin PC, Hux JE, Laupacis A. Epidural anaesthesia and survival after intermediate-to-high risk non-cardiac surgery: a population-based cohort study. Lancet. 2008;372(9638):562-9.

20. Cameron CM, Scott DA, McDonald WM, Davies MJ. A review of neuraxial epidural morbidity: experience of more than 8,000 cases at a single teaching hospital. Anesthesiology. 2007;106(5): 997-1002 Epub 2007/04/26.

21. Capdevila X, Pirat P, Bringuier S, Gaertner E, Singelyn F, Bernard $\mathrm{N}$, et al. Continuous peripheral nerve blocks in hospital wards after orthopedic surgery: a multicenter prospective analysis of the quality of postoperative analgesia and complications in 1,416 patients. Anesthesiology. 2005;103(5):1035-45.

22. Orebaugh SL, Kentor ML, Williams BA. Adverse outcomes associated with nerve stimulator-guided and ultrasound-guided peripheral nerve blocks by supervised trainees: update of a singlesite database. Reg Anesth Pain Med. 2012;37(6):577-82 Epub 2012/09/22.

23. Sites BD, Taenzer AH, Herrick MD, Gilloon C, Antonakakis J, Richins $\mathrm{J}$, et al. Incidence of local anesthetic systemic toxicity and postoperative neurologic symptoms associated with 12,668 ultrasound-guided nerve blocks: an analysis from a prospective clinical registry. Reg Anesth Pain Med. 2012;37(5):478-82.

24. Jacob AK, Mantilla CB, Sviggum HP, Schroeder DR, Pagnano MW, Hebl JR. Perioperative nerve injury after total hip arthroplasty: regional anesthesia risk during a 20-year cohort study. Anesthesiology. 2011;115(6):1172-8.

25. - Jacob AK, Mantilla CB, Sviggum HP, Schroeder DR, Pagnano MW, Hebl JR. Perioperative nerve injury after total knee arthroplasty: regional anesthesia risk during a 20-year cohort study. Anesthesiology. 2011;114(2):311-7. Epub 2011/01/18. This study indicated that the employment of regional anesthesia for total knee arthroplasty did not increase the risk of perioperative nerve injury. Recruitment of patients having one surgical procedure from a single hospital removed some of the clinical confounders.

26. Sviggum HP, Jacob AK, Mantilla CB, Schroeder DR, Sperling JW, Hebl JR. Perioperative nerve injury after total shoulder arthroplasty: assessment of risk after regional anesthesia. Reg Anesth Pain Med. 2012;37(5):490-4.

27. McNeil JJ, Evans SM, Johnson NP, Cameron PA. Clinical-quality registries: their role in quality improvement. Med $\mathrm{J}$ Aust. 2010;192(5):244-5.

28. Bufalino VJ, Masoudi FA, Stranne SK, Horton K, Albert NM, Beam C, et al. The American Heart Association's recommendations for expanding the applications of existing and future clinical registries: a policy statement from the American Heart Association. Circulation. 2011;123(19):2167-79.

29. Mangano DT, Tudor IC, Dietzel C. The risk associated with aprotinin in cardiac surgery. N Engl J Med. 2006;354(4):353-65 Epub 2006/01/27.

30. AQI. Anaesthesia Quality Institute. 2013. http://www.aqihq.org. Accessed 15 July 2013.

31. Barrington MJ, Watts SA, Gledhill SR, Thomas RD, Said SA, Snyder GL, et al. Preliminary results of the Australasian Regional Anaesthesia Collaboration: a prospective audit of more than 7000 peripheral nerve and plexus blocks for neurologic and other complications. Reg Anesth Pain Med. 2009;34(6):534-41.

32. Barrington MJ, Kluger R. Ultrasound guidance reduces the risk of local anesthetic systemic toxicity following peripheral nerve blockade. Reg Anesth Pain Med. 2013;38(4):289-97.
33. Antonakakis JG, Ting PH, Sites B. Ultrasound-guided regional anesthesia for peripheral nerve blocks: an evidence-based outcome review. Anesthesiol Clin. 2011;29(2):179-91.

34. Gelfand HJ, Ouanes JP, Lesley MR, Ko PS, Murphy JD, Sumida $\mathrm{SM}$, et al. Analgesic efficacy of ultrasound-guided regional anesthesia: a meta-analysis. J Clin Anesth. 2011;23(2):90-6 Epub 2011/03/08.

35. Abrahams MS, Aziz MF, Fu RF, Horn JL. Ultrasound guidance compared with electrical neurostimulation for peripheral nerve block: a systematic review and meta-analysis of randomized controlled trials. Br J Anaesth. 2009;102(3):408-17.

36. Hocking G, Mitchell $\mathrm{CH}$. Optimizing the safety and practice of ultrasound-guided regional anesthesia: the role of echogenic technology. Curr Opin Anaesthesiol. 2012;25(5):603-9.

37. Foxall GL, Hardman JG, Bedforth NM. Three-dimensional, multiplanar, ultrasound-guided, radial nerve block. Reg Anesth Pain Med. 2007;32(6):516-21.

38. Bedforth NM. New technologies in nerve location. Anaesthesia. 2010;65(Suppl 1):13-21.

39. Cash CJ, Sardesai AM, Berman LH, Herrick MJ, Treece GM, Prager RW, et al. Spatial mapping of the brachial plexus using three-dimensional ultrasound. Br J Radiol. 2005;78(936):1086-94.

40. Neal JM, Wedel DJ. Ultrasound guidance and peripheral nerve injury: is our vision as sharp as we think it is? Reg Anesth Pain Med. 2010;35(4):335-7 Epub 2010/07/01.

41. AAGBI. Recommendations for standards of monitoring during anaesthesia and recovery. The Association of Anaesthestists of Great Britain and Ireland; 2007.

42. AAGBI. The Anaesthesia Team. The Association of Anaesthetists of Great Britain and Ireland; 2010.

43. AAGBI. Management of severe local anaesthetic toxicity. The Association of Anaesthetists of Great Britain and Ireland; 2010.

44. ASRA. Checklist for Treatment of Local Anesthetic Systemic Toxcity. American Society for Regional Anesthesia and Pain Medicine; 2011.

45. Hebl JR, Dilger JA, Byer DE, Kopp SL, Stevens SR, Pagnano $\mathrm{MW}$, et al. A pre-emptive multimodal pathway featuring peripheral nerve block improves perioperative outcomes after major orthopedic surgery. Reg Anesth Pain Med. 2008;33(6):510-7.

46. Duncan CM, Hall Long v, Warner DO, Hebl JR. The economic implications of a multimodal analgesic regimen for patients undergoing major orthopedic surgery: a comparative study of direct costs. Reg Anesth Pain Med. 2009;34(4):301-7 Epub 2009/07/04.

47. WHO. WHO surgical safety checklist. World Health Organization; 2008.

48. Walker IA, Reshamwalla S, Wilson IH. Surgical safety checklists: do they improve outcomes? Br J Anaesth. 2012;109(1): 47-54 Epub 2012/06/01.

49. Haynes AB, Weiser TG, Berry WR, Lipsitz SR, Breizat AH, Dellinger EP, et al. A surgical safety checklist to reduce morbidity and mortality in a global population. $\mathrm{N}$ Engl $\mathrm{J}$ Med. 2009;360(5):491-9.

50. Haugen AS, Softeland E, Eide GE, Sevdalis N, Vincent CA, Nortvedt MW, et al. Impact of the World Health Organization's Surgical Safety Checklist on safety culture in the operating theatre: a controlled intervention study. Br J Anaesth. 2013;110(5): 807-15.

51. Allard J, Bleakley A, Hobbs A, Coombes L. Pre-surgery briefings and safety climate in the operating theatre. BMJ Qual Saf. 2011;20(8):711-7.

52. NUH. Stop before you Block Campaign. Safe Anaesthesia Liason Group, Nottingham University Hospitals, Regional Anaesthesia United Kingdom; 2011.

53. SALG. Wrong site blocks during surgery. Safe anaesthesia Liaison group; 2011. 
54. Marhofer P, Willschke H, Kettner S. Current concepts and future trends in ultrasound-guided regional anesthesia. Curr Opin Anaesthesiol. 2010;23(5):632-6.

55. Sites BD, Chan VW, Neal JM, Weller R, Grau T, KoscielniakNielsen ZJ, et al. The American Society of Regional Anesthesia and Pain Medicine and the European Society Of Regional Anaesthesia and Pain Therapy Joint Committee recommendations for education and training in ultrasound-guided regional anesthesia. Reg Anesth Pain Med. 2009;34(1):40-6.

56. Helwani MA, Saied NN, Asaad B, Rasmussen S, Fingerman ME. The current role of ultrasound use in teaching regional anesthesia: a survey of residency programs in the United States. Pain Med. 2012;13(10):1342-6.

57. - Grottke O, Ntouba A, Ullrich S, Liao W, Fried E, Prescher A, et al. Virtual reality-based simulator for training in regional anaesthesia. Br J Anaesth. 2009;103(4):594-600. Epub 2009/08/ 27. Describes the development and use of partial-task simulators for education and assessment of performance. Simulation provides a method of performing techniques before performing on patients.

58. Moore DL, Ding L, Sadhasivam S. Novel real-time feedback and integrated simulation model for teaching and evaluating ultrasound-guided regional anesthesia skills in pediatric anesthesia trainees. Paediatr Anaesth. 2012;22(9):847-53.

59. - Neal JM, Hsiung RL, Mulroy MF, Halpern BB, Dragnich AD, Slee AE. ASRA checklist improves trainee performance during a simulated episode of local anesthetic systemic toxicity. Reg Anesth Pain Med. 2012;37(1):8-15. Epub 2011/12/14.
Management of rare events can be learned using high-fidelity simulation and aided by checklists. In this randomized study, the use of a checklist significantly improved the trainees' medical management and nontechnical performance during an episode of severe local anesthetic systemic toxicity.

60. Barrington MJ, Wong DM, Slater B, Ivanusic JJ, Ovens M. Ultrasound-guided regional anesthesia: how much practice do novices require before achieving competency in ultrasound needle visualization using a cadaver model. Reg Anesth Pain Med. 2012;37(3):334-9.

61. RCOA. Direct Observation of Procedural Skills. Royal College of Anaethetists; 2010.

62. Gaba DM. Anaesthesiology as a model for patient safety in health care. BMJ. 2000;320(7237):785-8 Epub 2000/03/17.

63. Wu CL, Fleisher LA. Outcomes research in regional anesthesia and analgesia. Anesth Analg. 2000;91(5):1232-42.

64. Wu CL, Naqibuddin M, Fleisher LA. Measurement of patient satisfaction as an outcome of regional anesthesia and analgesia: a systematic review. Reg Anesth Pain Med. 2001;26(3):196-208.

65. Gornall BF, Myles PS, Smith CL, Burke JA, Leslie K, Pereira MJ, et al. Measurement of quality of recovery using the QoR-40: a quantitative systematic review. Br J Anaesth. 2013;111:161-9.

66. RCOA. Anaesthetic choices for hip or knee replacement. The Royal College of Anaesthetists, The Association of Anaesthetists of Great Britain and Ireland; 2008.

67. Barrington MJ. International registries of regional anesthesia: are we ready to collaborate in virtual departments of anesthesiology? Reg Anesth Pain Med. 2012;37(5):467-9. 